\title{
Automated procedure to determine the thermodynamic stability of a material and the range of chemical potentials necessary for its formation relative to competing phases and compounds
}

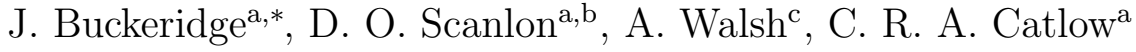 \\ ${ }^{a}$ University College London, Kathleen Lonsdale Materials Chemistry, Department of \\ Chemistry, 20 Gordon Street, London WC1H 0AJ, United Kingdom \\ ${ }^{b}$ Diamond Light Source Ltd., Diamond House, Harwell Science and Innovation Campus, \\ Didcot, Oxfordshire OX11 ODE, United Kingdom \\ ${ }^{c}$ Centre for Sustainable Chemical Technologies and Department of Chemistry, University \\ of Bath, Claverton Down, Bath BA2 7AY, United Kingdom
}

\begin{abstract}
We present a simple and fast algorithm to test the thermodynamic stability and determine the necessary chemical environment for the production of a multiternary material, relative to competing phases and compounds formed from the consituent elements. If the material is found to be stable, the region of stability, in terms of the constituent elemental chemical potentials, is determined from the intersection points of hypersurfaces in an $(n-1)$-dimensional chemical potential space, where $n$ is the number of atomic species in the material. The input required is the free energy of formation of the material itself, and that of all competing phases. Output consists of the result of the test of stability, the intersection points in the chemical potential space and the competing phase to which they relate, and, for two- and three-dimensional spaces, a file which may be used for visualization of the stability region. We specify the use of the program by applying it to a ternary and quaternary system. The algorithm automates essential analysis of the thermodynamic stability of a material. This analysis consists of a process which is lengthy for ternary materials, and becomes much more complicated when studying
\end{abstract}

\footnotetext{
${ }^{*}$ Corresponding author.

E-mail address: j.buckeridge@ucl.ac.uk
} 
materials of four or more consituent elements, which are of increased interest in recent years for technological applications such as energy harvesting and optoelectronics. The algorithm will therefore be of great benefit to the theoretical and computational study of such materials.

Keywords: Thermodynamic stability, Chemical potential, Materials design, Defect formation analysis

\section{PROGRAM SUMMARY}

Manuscript Title: Automated procedure to determine the thermodynamic stability of a material and the range of chemical potentials necessary for its formation relative to competing phases and compounds

Authors: J. Buckeridge, D. O. Scanlon, A. Walsh, C. R. A. Catlow

Program Title: CPLAP

Journal Reference:

Catalogue identifier:

Licensing provisions: none

Programming language: FORTRAN 90

Computer: Any computer with a FORTRAN 90 compiler

Operating system: Any OS with a FORTRAN 90 compiler

RAM: 2 megabytes

Number of processors used: one

Keywords: Thermodynamic stability, chemical potential, materials design, defect formation analysis

Classification: 16.1 Structure and properties, 23 Statistical Physics and Thermodynamics

Nature of problem:

To test the thermodynamic stability of a material with respect to competing phases and standard states of the constituent atomic species and, if stable, determine the range of chemical potentials consistent with its synthesis.

Solution method:

Assume that the formation of the material of interest occurs, rather than that of competing phases and standard states of the constituent elemental species. From this assumption derive a series of conditions on the elemental chemical potentials. Convert these conditions to a system of $m$ linear equations with $n$ unknowns, where $m>n$. Solve all combinations of $n$ linear equations, and test which solutions are compatible with the conditions on the chemical potentials. If none are, the system is unstable. Otherwise, the compatible results define boundary points of the stability region within the space spanned by the chemical potentials. Restrictions: 
The material growth environment is assumed to be in thermal and diffusive equilibrium.

Additional comments:

For two- and three-dimensional spaces spanned by the chemical potentials, files are produced for visualization of the stability region (if it exists).

Running time: Less than one second.

\section{Introduction}

Over the past few decades, there has been considerable growth in the development of advanced materials for energy harvesting and transparent electronics applications. [1, 2, 3, 4] At present, two of the greatest challenges facing the optoelectronics industry are the production of stable and economically viable $p$-type materials, $[5,6]$ and the replacement of rare or inaccessible components such as indium with more earth-abundant elements. [7, 8, 9, 10] This has led to increased interest in more exotic materials, consisting of ternary, $[11,12,13]$ quaternary, $[14,15,16]$ and quinternary $[17,18,19]$ systems. These materials are also of increased interest for applications in batteries [20] and solid state electrochemistry. [21] Having a large number of elements in a compound offers a greater degree in chemical freedom, where the tuning of properties of interest, such as band gaps, can be performed by varying the composition.

Instrumental in this research is the theoretical prediction of material properties, using various computational approaches, e.g. density functional theory (DFT) and methods based on interatomic potentials. [22] A key consideration when predicting materials appropriate for particular applications is the thermodynamical stability of the system, as stable materials present far fewer technological challenges when incorporated into devices. [23, 24] It is of great interest to predict the range of chemical potentials of the component elemental species over which the target phase is stable, rather than the elemental species themselves or competing phases, as this gives an indication the chemical environment necessary for the synthesis of that phase. Indeed, in order to predict the stability of a material, one needs to compare its free energy with that of all competing phases, including those consisting of subsets of the elemental species in the material. [25] The standard procedure [25, 26] is to calculate all relevant free energies at the athermal limit, under the assumption of thermodynamic equilibrium. Assuming that the material is 
thermodynamically stable, rather than the competing phases, leads to a set of conditions on the elemental chemical potentials, from which one can work out the stability range (if it exists). For binary systems, where the number of independent variables is one, the procedure is trivial. For ternary systems, though the calculation is still straightforward, if there are many competing phases, the exercise can become tedious. For quaternary or higher order systems, the calculation of the stability region becomes quite involved, as there are typically a large number of competing phases to consider, and three or more independent variables. It is evident that an automated process to perform these tasks would be of great benefit to theoriticians working on these problems.

Consideration of the chemical potential landscape within which a material forms is also crucial when predicting the nature and concentration of defects. The synthesis of a material in different conditions can mean that the formation of different defects becomes favorable. Calculations of defect formation energies, which depend on the chemical potentials, provide useful information to experimentalists wishing to produce a material with a particular defect-related property. For example, to produce a material with significant concentrations of a $p$-type donor incorporated during the growth process, it is necessary to know which chemical environment favors the formation of that particular donor defect. Knowledge of the full range of elemental chemical potentials within which the material is stable is required, in order to predict where in that range the formation of the $p$-type donor defect is favored. It is therefore necessary to work out accurately the stability region in the chemical potential space - not carrying out this procedure correctly can lead to unphysical predictions of defect formation energies. [27, 28] We stress that this type of analysis is limited to growth conditions where the assumption of thermodynamic equilibrium is reasonable.

In this paper, we present a simple, fast and effective algorithm to determine the range of the elemental chemical potentials within which the formation of a stoichiometric material will be favorable, in comparison to the formation of competing phases. If there is no range, then the material is not thermodynamically stable within the specified environment. The algorithm works by first reading in the free energy of formation of the material itself and that of the competing phases, which must be provided by the user. Setting the condition that the material is, in principle, stable constrains the values of the elemental chemical potentials, effectively reducing the number of independent variables by one, meaning that the space spanned by the elemental 
chemical potentials is $(n-1)$-dimensional, where $n$ is the total number of elements in the material. The condition that the competing phases do not form provides further conditional relations among the independent variables. A set of linear equations, corresponding to the set of all conditions on the independent variables, is constructed. All possible combinations of the linear equations in the set are solved in order to find their intersection points. The intersection points are then checked to determine which ones satisfy every condition (if none do the system is not thermodynamically stable). Those that do form the corner points of the region of stability in the chemical potential space. The algorithm is based on the fact that each competing phase and standard state effectively defines a hypersurface in the elemental chemical potential space, and the region bounded by these hypersurfaces corresponds to the region of values of chemical potentials in which the material will be stable. The elemental chemical potentials are given with respect to their standard states, i.e. we set the energy that the element has (per atom) in its standard state as the zero of chemical potential for that element. The algorithm requires that the energy of formation of the material and each competing phase is calculated (or measured) prior to execution. For an in silico study, it is therefore of great importance that the user searches the chemical databases (such as the Inorganic Crystal Structure Database [29]) extensively, and calculates the energy of all phases and limiting compounds using the same level of theory. [30, 31, 26, 25, 1] We have incorporated the algorithm in a FORTRAN program called 'Chemical Potential Limits Analysis Program' (CPLAP) which we have made available online. [32, 33] For convenience, if the chemical potential space is two-dimensional (2D) or threedimensional (3D), the program produces files that can be used as input to GNUPLOT [34] and MATHEMATICA, [35] to visualize the region of stability. An option to fix the value of a particular chemical potential is available, which effectively reduces the dimensionality by one.

The rest of the paper is structured as follows: In Sec. 2 we discuss the relevant theory on which the algorithm is based; in Sec. 3 we present the algorithm; in Sec. 4 we demonstrate how the program works using a ternary and quaternary system as examples; and in Sec. 5 we summarize our work. All the figures in this work, apart from the flowcharts, have been produced using GNUPLOT, from the output from CPLAP. 


\section{Theory}

The fundamental assumption, upon which analysis of the chemical potential landscape in which a material forms is based, is that the combined system in the growth environment is in thermodynamic equilibrium. To illustrate the necessary theory, we consider a binary system $A_{m} B_{n}$, which forms via the reaction:

$$
m A+n B \leftrightarrow A_{m} B_{n},
$$

at constant pressure and temperature. The formation of $A_{m} B_{n}$ competes with the phase $A_{p} B_{q}$. The procedure is then to assume that $A_{m} B_{n}$ forms, rather than $A_{p} B_{q}$ or the standard states of $A$ and $B$, and see if this leads to a contradiction.

We recall that the chemical potential $\mu_{\alpha}$ of species or compound $\alpha$ is defined as

$$
\mu_{\alpha}=\left(\frac{\partial G}{\partial N_{\alpha}}\right)_{p, T},
$$

where $G$ is the Gibbs free energy of the system $(G=U-T S+p V, U$ is the internal energy, $T$ is the temperature, $S$ is the entropy, $p$ is the pressure, and $V$ is the volume) and $N_{\alpha}$ is the number of particles of species or compound $\alpha$.

We first consider the chemical potential of individual species in the compound $A_{m} B_{n}$ (i.e. $A$ and $B$ ). We denote the chemical potential of species $\alpha$ in its standard state as $\mu_{\alpha}^{\mathrm{S}}$. We would now like to refer the elemental chemical potentials $\mu_{\alpha}$ to their respective $\mu_{\alpha}^{\mathrm{S}}$, i.e. we set

$$
\mu_{\alpha}=\mu_{\alpha}^{\mathrm{T}}-\mu_{\alpha}^{\mathrm{S}}
$$

where $\mu_{\alpha}^{\mathrm{T}}$ is the chemical potential of species $\alpha$ that shares a common reference with $\mu_{\alpha}^{\mathrm{S}}$. We do this for convenience; by determining the $\mu_{\alpha}^{\mathrm{S}}$ in a consistent manner, we will automatically obtain a common reference for all elemental chemical potentials. We note that, when calculating formation energies that depend on the chemical potentials, $\mu_{\alpha}^{\mathrm{T}}=\mu_{\alpha}^{\mathrm{S}}+\mu_{\alpha}$ should be used. In order to avoid formation of the standard states of $A$ and $B$, we must have

$$
\mu_{\alpha} \leq 0
$$

placing an upper bound on each elemental chemical potential.

We now consider all species involved in the reaction given in Eqn. 1, so that $\alpha=A, B, A_{m} B_{n}$, and follow the analysis given in Ref. [36]. Under the 
assumption of constant $p$ and $T$, the differential $d G$ in the Gibbs free energy is given by:

$$
d G=\sum_{\alpha} \mu_{\alpha}^{\mathrm{T}} d N_{\alpha} .
$$

As $d N_{\alpha}$ is proportional to the coefficient $i_{\alpha}$ in the reaction given by Eqn. 1 $\left(i_{\alpha}=m\right.$ for $\alpha=A, i_{\alpha}=n$ for $\alpha=B, i_{\alpha}=-1$ for $\left.\alpha=A_{m} B_{n}\right)$, it can be written as $d N_{\alpha}=i_{\alpha} d N$, where $d N$ is the number of occurences of the reaction in Eqn. 1. We can therefore write

$$
d G=\left(\sum_{\alpha} i_{\alpha} \mu_{\alpha}^{\mathrm{T}}\right) d N
$$

At equilibrium, ${ }^{1} d G=0$, implying that

$$
\sum_{\alpha} i_{\alpha} \mu_{\alpha}^{\mathrm{T}}=0
$$

from which we obtain (remembering $i_{A_{m} B_{n}}=-1$ ):

$$
m \mu_{A}+n \mu_{B}=\mu_{A_{m} B_{n}}=\Delta G_{f}\left[A_{m} B_{n}\right] ;
$$

here $\Delta G_{f}[X]=\Delta H_{f}[X]-T \Delta S$ is the Gibbs free energy of formation of compound $X$ with respect to the standard states of its constituent elements, $H_{f}[X]$ is the enthalpy of formation of $X$, and $\Delta S$ is the change in entropy. For crystalline systems with low levels of disorder, a good approximation is to set $\Delta S=0$, so that $\Delta G_{f}[X]=\Delta H_{f}[X]$. Under this approximation we can set the chemical potentials of $A$ and $B$ in their standard states equal to the total energy (per atom) of the standard states. Calculating all total energies, including those required to determine $\Delta H_{f}[X]$, in a consistent manner ensures all chemical potentials have a common reference. Although it is possible to include vibrational entropic effects using, for example, the quasiharmonic approximation, and configurational entropic effects for disordered systems, in the remainder of this paper we assume that the approximation $\Delta G_{f}[X]=\Delta H_{f}[X]$ applies. Eqn. 8 now becomes:

$$
m \mu_{A}+n \mu_{B}=\mu_{A_{m} B_{n}}=\Delta H_{f}\left[A_{m} B_{n}\right],
$$

\footnotetext{
${ }^{1}$ Once equilibrium is reached, the reaction will not proceed further; therefore there will not be any further change in the thermal average values of the concentrations. This implies that, given the volume at equilibrium, Eqn. 7 will be valid when $V$ and $T$ are specified instead of $p$ and $T$, as was our initial assumption. See Ref. [36]
} 
effectively constraining our mathematical problem, so that one chemical potential can be written in terms of the other, i.e. the number of independent variables is one. For a binary system, therefore, the chemical potential space is one-dimensional (1D), spanned by the one independent variable.

Combining Eqns. 4 and 9 and taking $\mu_{A}$ to be the independent variable, we find that:

$$
\frac{\Delta H_{f}\left[A_{m} B_{n}\right]}{m} \leq \mu_{A} \leq 0
$$

with $\mu_{B}$ being determined for each value of $\mu_{A}$ from Eqn. 9. It follows then that the boundary $\mu_{A}=0$ corresponds to $A$-rich $B$-poor growth conditions, and the boundary $\mu_{A}=\Delta H_{f}\left[A_{m} B_{n}\right] / m$ corresponds to $B$-rich $/ A$-poor growth conditions. Eqn. 10 defines the stability region (a line segment) in the $1 \mathrm{D}$ chemical potential space spanned by $\mu_{A}$.

We now include in our calculation the competing phase $A_{p} B_{q}$. The assumption that $A_{p} B_{q}$ does not form leads to the following condition:

$$
p \mu_{A}+q \mu_{B} \leq \mu_{A_{p} B_{q}}=\Delta H_{f}\left[A_{p} B_{q}\right] .
$$

Combining this with Eqn. 9 provides the following limits:

$$
\begin{aligned}
\left(p-\frac{q m}{n}\right) \mu_{A} & \leq \Delta H_{f}\left[A_{p} B_{q}\right]-\frac{q}{n} \Delta H_{f}\left[A_{m} B_{n}\right] \\
\left(q-\frac{p n}{m}\right) \mu_{B} & \leq \Delta H_{f}\left[A_{p} B_{q}\right]-\frac{p}{m} \Delta H_{f}\left[A_{m} B_{n}\right] .
\end{aligned}
$$

If these limits are inconsistent with Eqn. 4 then $A_{m} B_{n}$ is unstable with respect to the formation of $A_{p} B_{q}$. If they are consistent, then they effectively reduce the range given in Eqn. 10, i.e. they reduce the extent of the stability region. The addition of more competing phases will further restrict the stability region, which will (if it exists) consist of a line segment in the $1 \mathrm{D}$ space spanned by $\mu_{A}$, with corresponding values of $\mu_{B}$ derived from Eqn. 9 . This solves the case of a binary system.

We now consider a ternary system, to demonstrate the generalization of the process as one increases the dimensionality of the chemical potential space. We consider the system $A_{m} B_{n} C_{p}$, whose formation competes with the phases $A_{q} B_{r}$ and $A_{s} B_{t} C_{v}$.

Corresponding to Eqn. 9, the assumption that $A_{m} B_{n} C_{p}$ forms in an equilibrium reaction with the constituent elements' standard phases provides the constraint:

$$
m \mu_{A}+n \mu_{B}+p \mu_{C}=\mu_{A_{m} B_{n} C_{p}}=\Delta H_{f}\left[A_{m} B_{n} C_{p}\right]
$$


allowing us to express one of the chemical potentials, say $\mu_{C}$, in terms of the other two, leaving two independent variables $\mu_{A}$ and $\mu_{B}$ spanning a $2 \mathrm{D}$ chemical potential space. Allowing $\mu_{C}$ to adopt its maximum bounded value of zero (see Eqn. 4) gives the following condition on $\mu_{A}$ and $\mu_{B}$ :

$$
m \mu_{A}+n \mu_{B} \geq \Delta H_{f}\left[A_{m} B_{n} C_{p}\right] .
$$

Combining Eqns. 4 and 13 gives the following conditions on the chemical potentials:

$$
\mu_{i} \geq \Delta H_{f}\left[A_{m} B_{n} C_{p}\right] / i_{\alpha},
$$

where $i_{\alpha}$ stands for either $m, n$, or $p$, whichever is appropriate.

Assuming the competing phases do not form leads to the conditions:

$$
\begin{aligned}
q \mu_{A}+r \mu_{B} & \leq \mu_{A_{q} B_{r}}=\Delta H_{f}\left[A_{q} B_{r}\right] \\
s \mu_{A}+t \mu_{B}+v \mu_{C} & \leq \mu_{A_{s} B_{t} C_{v}}=\Delta H_{f}\left[A_{s} B_{t} C_{v}\right] .
\end{aligned}
$$

Using Eqn. 13 to eliminate $\mu_{C}$ from Eqn. 17, we see that Eqns. 4, 14, 15, 16, and 17 define conditional relations on a $2 \mathrm{D}$ plane formed by $\mu_{A}$ and $\mu_{B}$. If there does not exist a region in the $2 \mathrm{D}$ plane that conforms to every condition, then the system is not thermodynamically stable. Otherwise, we have a region of stability. One method of determining if this is the case is to set the inequality signs in Eqns. 4, 14, 15, 16, and 17 to equality signs, giving a series of linear equations with two unknowns. These linear equations define lines on the $2 \mathrm{D}$ plane formed by $\mu_{A}$ and $\mu_{B}$. Their intersection points can be determined by solving the appropriate combinations of the linear equations. Those that then simultaneously satisfy the conditions given by Eqns. 4, 14, 15, 16 and 17 (if any) will bound the region of stability. The result will be a $2 \mathrm{D}$ stability region in the plane defined by $\mu_{A}$ and $\mu_{B}$, with the corresponding value of $\mu_{C}$ at each point in the stability region determined from Eqn. 13. Graphically, one can display this solution as a 2D plot in the space spanned by $\mu_{A}$ and $\mu_{B}$, with the corresponding values of $\mu_{C}$ given at points of interest.

The generalization of this procedure to systems with larger number of constituent elements is as follows. For a system with $n$ constituent elements, we will have $n-1$ independent variables. The higher dimensional analogues of Eqns. 4, 14, and 15 provide $2 n-1$ linear equations (which correspond to hypersurfaces in the $(n-1)$-dimensional space), and each competing phase provides an additional linear equation. We therefore have a minimum of 
$2 n-1$ linear equations with $n-1$ unknowns. Mathematically, the solution is trivial, as it only involves solving different combinations of the linear equations and checking which solutions are compatible with a series of conditional statements. In practice, however, if we have $m$ competing phases there are ${ }^{2 n+m-1} C_{n-1}$ combinations to consider, and carrying out the procedure can be quite time consuming and error-prone. This is the reason we have developed a program to automate it.

\section{Algorithm}

Input to the program consists of the number of species in the compound of interest, the names and stoichiometry of the species, and the free energy of formation of the compound. One must also input the total number (if any) of competing phases, and, for each one, the number of species, the names and stoichiometry of each species, and the free energy of formation of that competing phase. The input can be provided via a file, or interactively while running the program.

The user must specify which elemental chemical potential is to be set as the dependent variable. We note here that the procedure carried out by the program can, in principle, be performed without any dependent variable set. If this is done, however, only the intersection points with the hypersurface corresponding to the compound of interest are viable solutions, since, by not setting a dependent variable, the constraint given by Eqns. 9 or 13 (or the higher-dimensional analogue) is assumed no longer to apply, and instead effectively the equality sign is replaced by a 'greater than or equals to' sign (i.e. the assumption that the reaction in Eqn. 1 is in equilibrium no longer holds). Only those results that are consistent with the constraint are actual solutions of the problem at hand. So, though more intersection points may be found when no dependent variable is set, only those that intersect the hypersurface corresponding to the compound of interest are actual solutions. If no dependent variable is set, the program warns the user of this fact, and how to interpret the results. It is always preferable to set a dependent variable.

After reading in the input, the main algorithm begins (see Fig. 1). If the system is binary, the solution is relatively trivial. The program carries out the procedure as described in Sec. 2 for binary systems, which is to check that the limits imposed by the competing phases (Eqns. 12) are consistent with Eqn. 4 and the constraint (Eqn. 9), and, if they are, to return the line 
segment that defines the region of stability. The constraint is also returned as output. Note that this is a separate procedure from that used when the number of species is greater than two.

For ternary and higher-order systems a more complex algorithm is used. From the input, the program constructs a matrix of linear equations with $n-1$ unknowns, where $n$ is the number of species in the system. The compound of interest itself provides one linear equation (Eqn. 14, or its higher-dimensional analogue). Each independent variable then contributes two linear equations; one given by Eqn. 4, the other given by Eqn. 15, which means that there are, at a minimum, $2 n-1$ linear equations in the matrix. Additional equations are provided by the competing phases: one per phase. If there are $m$ competing phases, we therefore have, in total, $2 n+m-1$ linear equations. Once the matrix has been constructed, it is passed to a sorting routine which extracts every possible combination of $n-1$ equations from the $2 n+m-1$ total. This sorting routine is described in Appendix A. For each combination, the $n-1$ equations are solved using a standard $L U$ decomposition and back-substitution method, [37] if a solution exists. In this way a series of intersection points are found (redundencies are checked for, and removed). Each intersection point is tested to see if it obeys simultaneously all the conditions on the elemental chemical potentials (Eqns. 4, 14, 15, 16, and 17 or higher-dimensional analogues). If none do, the system is not thermodynamically stable. Otherwise, those that do correspond to corner points in the stability region. The output is then sent to file, consisting of the limiting conditions applied, the resulting intersection points (with, for each one, the corresponding value of the dependent variable), and a list composed of each competing phase, with its corresponding linear equation and intersection points (if any).

An option is provided to print to file a grid of points within the stability region, with the grid density provided by the user. Such a grid of values may be useful for demonstrating the variation of the formation energy of a particular defect as the elemental chemical potentials are varied; for this the user would be required to calculate the formation energy at each grid point. If the chemical potential space is $2 \mathrm{D}$ or $3 \mathrm{D}$, the program outputs a file which may be loaded directly into GNUPLOT, and text which may be pasted into a notebook in MATHEMATICA, to produce a plot of the stability region, which will be useful for visualization of results. In addition, for $2 \mathrm{D}$ chemical potential spaces, a text file is produced which contains the necessary data to plot the lines in the chemical potential space corresponding to the material 
of interest and its competing phases.

It is possible to restart a run from a previous calculation. Options are then available to set a different chemical potential as the dependent variable, to provide additional competing phases not considered in the original run, or to set a chemical potential to a particular value (effectively reducing the dimensionality of the chemical potential space by one). The latter option is not available for binary systems, as the solution is trivial.

We note that, in principle, the procedure could be extended to arbitrary pressure and temperature ranges by including thermodynamic potentials either from computations (using phonon frequency calculations and/or statistical mechanics) or thermochemical data. Such an extension is beyond the immediate scope of the present work.

Our approach should be compared with that of the CALPHAD code, [38, 39, 40] which is widely used in modelling phase diagrams of alloys over a range of temperature, pressure and composition. CALPHAD uses a model with adjustable parameters to describe the thermodynamic properties of each phase of a material, fitting the parameters to results from thermochemical and thermophysical studies stored in databases, and determines a consistent phase diagram using a wide range of data. The aim of our approach is different; it identifies the range of elemental chemical potentials over which a specified phase is stable.

\section{Examples}

\subsection{Ternary system}

As our first example of the application of our program, we consider the system $\mathrm{BaSnO}_{3}$, [41] an indium-free transparent conducting oxide (TCO). The formation of $\mathrm{BaSnO}_{3}$ (in the cubic perovskite structure) occurs in competition with the phases $\mathrm{BaO}, \mathrm{SnO}, \mathrm{SnO}_{2}$, and $\mathrm{Ba}_{2} \mathrm{SnO}_{4}$, as determined by searching the Inorganic Crystal Structure Database [29] for systems consisting of combinations of the elements $\mathrm{Ba}, \mathrm{Sn}$, and O. Our aim here is to determine the ranges of chemical potentials in which stoichiometric $\mathrm{BaSnO}_{3}$ will form, using our program. The enthalpies of formation of the competing phases and the material itself have been calculated previously, [41] using DFT with the PBE0 $[42,43]$ hybrid functional (at the athermal limit). The values are presented in Table 1. These, and the stoichiometries of the relevant compounds, form the input to our program. The constraint on the 
elemental chemical potentials is (see Eqn. 13):

$$
\mu_{\mathrm{Ba}}+\mu_{\mathrm{Sn}}+3 \mu_{\mathrm{O}}=\mu_{\mathrm{BaSnO}_{3}}=-11.46 \mathrm{eV} .
$$

We set the chemical potential of $\mathrm{O}, \mu_{\mathrm{O}}$, as the dependent variable.

Table 1: Enthalpies of formation $\left(\Delta H_{f}\right)$ of $\mathrm{BaSnO}_{3}$ and its relevant competing phases. The values, which are taken from Ref. [41], were determined using DFT with the PBE0 hybrid functional.

\begin{tabular}{l|c|c|c}
\hline \hline System & $\Delta H_{f}(\mathrm{eV})$ & System & $\Delta H_{f}(\mathrm{eV})$ \\
\hline $\mathrm{BaSnO}_{3}$ & -11.46 & $\mathrm{Ba}_{2} \mathrm{SnO}_{4}$ & -17.13 \\
$\mathrm{BaO}$ & -5.14 & $\mathrm{SnO}$ & -2.54 \\
$\mathrm{SnO}_{2}$ & -5.29 & & \\
\hline \hline
\end{tabular}

After running the program, we find that the system is thermodynamically stable. Given that $\mathrm{BaSnO}_{3}$ forms, the limiting conditions that apply to the two independent variables $\mu_{\mathrm{Ba}}$ and $\mu_{\mathrm{Sn}}$ are (energies in $\mathrm{eV}$ ):

$$
\begin{aligned}
\mu_{\mathrm{Ba}}+\mu_{\mathrm{Sn}} & \geq-11.46, \\
2 \mu_{\mathrm{Ba}}-\mu_{\mathrm{Sn}} & \leq-5.52, \\
2 \mu_{\mathrm{Ba}}-\mu_{\mathrm{Sn}} & \leq-3.95 \\
-\mu_{\mathrm{Ba}}+2 \mu_{\mathrm{Sn}} & \leq 3.85, \\
\mu_{\mathrm{Ba}} & \leq 0 \\
\mu_{\mathrm{Sn}} & \leq 0 \\
\mu_{\mathrm{Ba}} & \geq-11.46, \\
\mu_{\mathrm{Sn}} & \geq-11.46 .
\end{aligned}
$$

We present the resulting intersection points bounding the stability region in Table 2, where we give the corresponding value of the dependent variable $\mu_{\mathrm{O}}$, and the competing phases to which the intersection points correspond. The stability region is plotted in Fig. 2. We note that, if we change which chemical potential is set as the dependent variable, we obtain the same results (as we must). The only difference will be in the appearance of the figure, as one of the axes will be changed to that of the new independent variable.

It is worth noting that if one of the competing phases, say $\mathrm{Ba}_{2} \mathrm{SnO}_{4}$ (which could easily be overlooked), is not included in the calculation, the 
Table 2: Intersection points bounding the stability region in the $2 \mathrm{D}$ chemical potential space spanned by the independent variables $\mu_{\mathrm{Ba}}$ and $\mu_{\mathrm{Sn}}$. The corresponding values of the dependent variable $\mu_{\mathrm{O}}$, and the relevant competing phases, are also given. All energies are in $\mathrm{eV}$.

\begin{tabular}{|c|c|c||c|c|}
\hline \hline & $\mu_{\mathrm{Ba}}$ & $\mu_{\mathrm{Sn}}$ & $\mu_{\mathrm{O}}$ & Competing phases \\
\hline $\mathrm{A}$ & -5.66 & -5.80 & 0.00 & $\mathrm{Ba}_{2} \mathrm{SnO}_{4}, \mathrm{BaSnO}_{3}$ \\
$\mathrm{~B}$ & -6.18 & -5.29 & 0.00 & $\mathrm{SnO}_{2}, \mathrm{BaSnO}_{3}$ \\
$\mathrm{C}$ & -2.76 & 0.00 & -2.90 & $\mathrm{Ba}_{2} \mathrm{SnO}_{4}$ \\
$\mathrm{D}$ & -3.53 & 0.00 & -2.64 & $\mathrm{SnO}_{2}$ \\
\hline \hline
\end{tabular}

resulting stability region (see Fig. 3) is approximately twice as extensive as that shown in Fig. 2, indicating the importance of taking into account all relevant competing phases. If one or more is left out, the analysis may be incorrect. Similarly, when calculating the total energies of the standard phases of the constituent elements, the correct ground-state of $\mathrm{O}_{2}$ (triplet spin configuration) must be used, as well as sufficient $k$-point sampling for the metallic standard phases.

As is discussed in Ref. [41], the most stable $n$-type intrinsic defect in $\mathrm{BaSnO}_{3}$ is the $\mathrm{O}$ vacancy $\left(\mathrm{V}_{\mathrm{O}}\right)$. The formation enthalpy $\Delta H_{f}\left[\mathrm{~V}_{\mathrm{O}}\right]$ of the (neutral) defect is determined from the reaction

$$
\mathrm{O}_{\mathrm{O}} \rightarrow \mathrm{V}_{\mathrm{O}}+\frac{1}{2} \mathrm{O}_{2}
$$

according to:

$$
\Delta H_{f}\left[V_{\mathrm{O}}\right]=\left(E^{D}-E^{H}\right)+E_{\mathrm{O}_{2}}+\mu_{\mathrm{O}},
$$

where $E^{H}$ is the energy of the stoichiometric host supercell, $E^{D}$ is the energy of a supercell containing the defect, and $E_{\mathrm{O}_{2}}$ is the energy per atom of $\mathrm{O}$ in its elemental $\left(\mathrm{O}_{2}\right.$ gas $)$ form, which we have set as the chemical potential of $\mathrm{O}$ in its standard state, $\mu_{\mathrm{O}}^{\mathrm{S}}$ (see Sec. 2). As can be seen, $\Delta H_{f}\left[\mathrm{~V}_{\mathrm{O}}\right]$ depends on $\mu_{\mathrm{O}}$. By printing a grid of points contained within the stability region, one obtains a list of points at which $\Delta H_{f}\left[\mathrm{~V}_{\mathrm{O}}\right]$ can be determined, which in turn can be used to demonstrate how the defect formation energy varies at the different possible growth conditions. We show the results of such a calculation in Fig. 4, where the variation in $\Delta H_{f}\left[\mathrm{~V}_{\mathrm{O}}\right]$ is shown within the stability region in the chemical potential space. We see that, unsurprisingly, Ba- and Sn-rich conditions favor its formation. It should be remembered that the defect concentration depends exponentially on this quantity. 


\subsection{Quaternary system}

We now discuss the application of CPLAP to the quaternary system LaCuOSe. This layered oxyselenide is a promising degenerate $p$-type wide bandgap semiconductor. [44, 45, 46, 15] With four species in the compound, we have a $3 \mathrm{D}$ chemical potential space. There are a large number of competing phases (22) to be taken into consideration, as determined by searching the Inorganic Crystal Structure Database [29] for systems consisting of combinations of the elements $\mathrm{La}, \mathrm{Cu}, \mathrm{O}$, and Se. We therefore have a much more complicated problem than for the ternary system $\mathrm{BaSnO}_{3}$, discussed in Sec. 4.1. This example demonstrates well the power of our program in analyzing the chemical potential ranges.

We have calculated the enthalpy of formation of the compound and its competing phases using DFT with the HSE06 [47] hybrid functional. Our purpose here is to discuss the ranges of chemical potentials consistent with the growth of the material, which can support future studies of its defect and materials physics. The calculated enthalpies of formation are shown in Table 3. These, along with the stoichiometries of the compounds, form the input to CPLAP.

Table 3: Enthalpies of formation $\left(\Delta H_{f}\right)$ of LaCuOSe and its relevant competing phases. The values were determined using DFT with the HSE06 hybrid functional.

\begin{tabular}{l|c|l|c}
\hline \hline System & $\Delta H_{f}(\mathrm{eV})$ & $\mathrm{System}$ & $\Delta H_{f}(\mathrm{eV})$ \\
\hline $\mathrm{LaCuOSe}$ & -9.55 & $\mathrm{CuSe}_{2}$ & -1.16 \\
$\mathrm{La}_{2} \mathrm{CuO}_{4}$ & -19.94 & $\mathrm{CuSe}_{2} \mathrm{O}_{5}$ & -6.54 \\
$\mathrm{CuLaO}_{2}$ & -10.60 & $\mathrm{La}_{2} \mathrm{SeO}_{2}$ & -16.27 \\
$\mathrm{La}_{2} \mathrm{O}_{3}$ & -17.70 & $\mathrm{La}_{2}\left(\mathrm{SeO}_{3}\right)_{3}$ & -27.94 \\
$\mathrm{La}_{3} \mathrm{Se}_{4}$ & -15.77 & $\mathrm{La}_{4} \mathrm{Se}_{3} \mathrm{O}_{4}$ & -33.16 \\
$\mathrm{LaCuSe}_{2}$ & -6.96 & $\mathrm{LaCu}_{2}$ & -2.13 \\
$\mathrm{LaSe}_{2}$ & -5.64 & $\mathrm{LaCu}_{5}$ & -4.52 \\
$\mathrm{LaSe}_{\mathrm{Ce}} \mathrm{Se}_{\mathrm{CuSe}}$ & -4.41 & $\mathrm{La}\left(\mathrm{CuO}_{2}\right)_{2}$ & -13.07 \\
$\mathrm{Cu}_{3} \mathrm{Se}$ & & $\mathrm{LaCuO}_{3}$ & -10.61 \\
$\mathrm{La}_{2} \mathrm{Cu}\left(\mathrm{SeO}_{3}\right)_{4}$ & -1.95 & $\mathrm{Se}_{2} \mathrm{O}_{5}$ & -3.37 \\
\hline \hline
\end{tabular}

The constraint on the chemical potentials is:

$$
\mu_{\mathrm{La}}+\mu_{\mathrm{Cu}}+\mu_{\mathrm{O}}+\mu_{\mathrm{Se}}=\mu_{\mathrm{LaCuOSe}}=-9.55 \mathrm{eV} .
$$


Table 4: Intersection points bounding the stability region in the 3D chemical potential space spanned by the independent variables $\mu_{\mathrm{La}}, \mu_{\mathrm{Cu}}$ and $\mu_{\mathrm{O}}$. The corresponding values of the dependent variable $\mu_{\mathrm{Se}}$, and the relevant competing phases, are also given. All energies are in $\mathrm{eV}$.

\begin{tabular}{|c|c|c|c|c|c|}
\hline & $\mu_{\mathrm{La}}$ & $\mu_{\mathrm{Cu}}$ & $\mu_{\mathrm{O}}$ & $\mu_{\mathrm{Se}}$ & Competing phases \\
\hline $\bar{A}$ & -5.78 & -1.18 & -2.59 & 0.00 & $\mathrm{LaCuSe}_{2}, \mathrm{Cu}_{3} \mathrm{Se}_{2}, \mathrm{LaCuOSe}$ \\
\hline B & -5.70 & -1.26 & -2.59 & 0.00 & $\mathrm{LaCuSe}_{2}, \mathrm{La}_{4} \mathrm{Se}_{3} \mathrm{O}_{4}, \mathrm{LaCuOSe}$ \\
\hline $\mathrm{C}$ & -6.77 & -1.18 & -1.60 & 0.00 & $\mathrm{Cu}_{3} \mathrm{Se}_{2}, \mathrm{La}_{2}\left(\mathrm{SeO}_{3}\right)_{3}, \mathrm{LaCuOSe}$ \\
\hline $\mathrm{D}$ & -6.67 & -1.26 & -1.62 & 0.00 & $\mathrm{La}_{2}\left(\mathrm{SeO}_{3}\right)_{3}, \mathrm{La}_{4} \mathrm{Se}_{3} \mathrm{O}_{4}, \mathrm{LaCuOSe}$ \\
\hline $\mathrm{E}$ & -6.62 & -1.00 & -1.49 & -0.44 & $\mathrm{CuLaO}_{2}, \mathrm{La}_{2} \mathrm{O}_{3}, \mathrm{La}_{2}\left(\mathrm{SeO}_{3}\right)_{3}$ \\
\hline $\mathrm{F}$ & -3.95 & -0.11 & -3.27 & -2.22 & $\mathrm{CuLaO}_{2}, \mathrm{La}_{2} \mathrm{O}_{3}, \mathrm{LaCu}_{5}$ \\
\hline G & -5.67 & -0.36 & -2.28 & -1.24 & $\mathrm{CuLaO}_{2}, \mathrm{Cu}_{2} \mathrm{Se}, \mathrm{Cu}_{3} \mathrm{Se}_{2}$ \\
\hline $\mathrm{H}$ & -4.60 & 0.00 & -3.00 & -1.95 & $\mathrm{CuLaO}_{2}, \mathrm{Cu}_{2} \mathrm{Se}$ \\
\hline $\mathrm{I}$ & -6.77 & -0.91 & -1.46 & -0.42 & $\mathrm{CuLaO}_{2}, \mathrm{Cu}_{3} \mathrm{Se}_{2}, \mathrm{La}_{2}\left(\mathrm{SeO}_{3}\right)_{3}$ \\
\hline $\mathrm{J}$ & -4.52 & 0.00 & -3.04 & -1.99 & $\mathrm{CuLaO}_{2}, \mathrm{LaCu}_{5}$ \\
\hline K & -5.77 & -1.11 & -2.05 & -0.62 & $\mathrm{La}_{2} \mathrm{O}_{3}, \mathrm{La}_{2} \mathrm{SeO}_{2}, \mathrm{La}_{4} \mathrm{Se}_{3} \mathrm{O}_{4}$ \\
\hline $\mathrm{L}$ & -3.23 & -0.26 & -3.75 & -2.32 & $\mathrm{La}_{2} \mathrm{O}_{3}, \mathrm{La}_{2} \mathrm{SeO}_{2}, \mathrm{LaCu}_{5}$ \\
\hline M & -6.51 & -1.19 & -1.56 & -0.29 & $\mathrm{La}_{2} \mathrm{O}_{3}, \mathrm{La}_{2}\left(\mathrm{SeO}_{3}\right)_{3}, \mathrm{La}_{4} \mathrm{Se}_{3} \mathrm{O}_{4}$ \\
\hline $\mathrm{N}$ & -2.96 & -0.56 & -4.31 & -1.72 & $\mathrm{La}_{3} \mathrm{Se}_{4}, \mathrm{LaCuSe}_{2}, \mathrm{La}_{2} \mathrm{SeO}_{2}$ \\
\hline $\mathrm{O}$ & -2.61 & -0.38 & -4.57 & -1.98 & $\mathrm{La}_{3} \mathrm{Se}_{4}, \mathrm{LaCuSe}_{2}, \mathrm{LaCu}_{5}$ \\
\hline $\mathrm{P}$ & -2.54 & -0.40 & -4.58 & -2.04 & $\mathrm{La}_{3} \mathrm{Se}_{4}, \mathrm{La}_{2} \mathrm{SeO}_{2}, \mathrm{LaCu}_{5}$ \\
\hline Q & -4.12 & -0.36 & -3.83 & -1.24 & $\mathrm{LaCuSe}_{2}, \mathrm{Cu}_{2} \mathrm{Se}, \mathrm{Cu}_{3} \mathrm{Se}_{2}$ \\
\hline $\mathrm{R}$ & -3.60 & -0.18 & -4.18 & -1.59 & $\mathrm{LaCuSe}_{2}, \mathrm{Cu}_{2} \mathrm{Se}, \mathrm{LaCu}_{5}$ \\
\hline $\mathrm{S}$ & -4.61 & -1.11 & -3.21 & -0.62 & $\mathrm{LaCuSe}_{2}, \mathrm{La}_{2} \mathrm{SeO}_{2}, \mathrm{La}_{4} \mathrm{Se}_{3} \mathrm{O}_{4}$ \\
\hline $\mathrm{T}$ & -4.52 & 0.00 & -3.08 & -1.95 & $\mathrm{Cu}_{2} \mathrm{Se}, \mathrm{LaCu}_{5}$ \\
\hline
\end{tabular}
the system is thermodynamically stable. As there are 29 limiting conditions on the independent variables, we do not list them here. We find 20 intersection points in the $3 \mathrm{D}$ chemical potential space spanned by $\mu_{\mathrm{La}}, \mu_{\mathrm{Cu}}$, and $\mu_{\mathrm{O}}$. They are presented, along with the corresponding values of $\mu_{\mathrm{Se}}$ and the relevant competing phases, in Table 4 . The 3D stability region is shown in Fig. 5. The relevant competing phases describe $2 \mathrm{D}$ surfaces in the $3 \mathrm{D}$ space, which are shown using colors in Fig. 5 (we note that, because GNUPLOT cannot plot surfaces parallel to the $z$-axis, we must represent such surfaces by placing a cross at their mid-point, as we do for the competing phase $\mathrm{LaCu}_{5}$ ).

We choose $\mu_{\text {Se }}$ as the dependent variable. Running the program, we find that

${ }_{425}$ If we are interested in, e.g., O-poor conditions, we can set $\mu_{\mathrm{O}}$ to a low 
value, say $-4 \mathrm{eV}$ (which is close to, but a little above its minimum value of $-4.58 \mathrm{eV}$ - see Table 4). Doing this reduces the dimensionality of the problem by one. The resulting stability region is a 2D 'slice' taken from the 3D stability region shown in Fig. 5 . We present this $2 \mathrm{D}$ stability region in Fig. 6, along with the relevant competing phases, which describe lines in the 2D chemical potential space.

Other sections of the stability region that may be of interest can be extracted easily by setting chemical potentials to particular values. The visualization of the resulting regions can be further modified by changing the dependent variable. This demonstrates the versatility of CPLAP in exploring the region of stability in the chemical potential space. To carry out these types of manipulations, in particular for a quaternary system such as LaCuOSe, 'by hand' can be quite time-consuming and error-prone. Once the calculation is set up, the region of stability can be explored easily and accurately, with visualization possible when the system is 2D or 3D. For quinternary (or higher order) systems, one has to set a chemical potential to a particular value before the stability region can be visualized (in 3D). The ease with which one can systematically explore the stability region using CPLAP will be of great benefit to the theoretical and computational study of systems consisting of 4 or more species.

\section{Conclusion}

In summary, we have described a simple and effective algorithm to determine the thermodynamical stability and range of chemical potentials consistent with the formation of a particular compound of interest, in comparison with the formation of competing phases and elemental forms of the constituent species. By assuming that the compound of interest forms in equilibrium, rather than competing phases and standard states, a set of conditions on the chemical potentials can be derived. These conditions can be interpreted as defining a region bounded by hyper-surfaces in an $(n-1)$ dimensional chemical potential space, where $n$ is the number of species in the system. Determining this region of stability gives the chemical potential landscape consistent with the production of the compound of interest. The algorithm works by reading in the energies of formation of the compound itself, and all competing phases, then constructing a matrix of linear equations, solving all possible combinations of the equations, and finding which solutions (if any) obey the conditions on the chemical potentials. We have 
incorporated the algorithm in a FORTRAN program (CPLAP). Options are available to set a chemical potential to a particular value, and to print a grid of points within the stability region. For 2D and 3D systems, files are produced to allow visualization of the results. We have demonstrated the effectiveness of the program using a ternary and quaternary system. We have also demonstrated the flexibility with which the program may be used to explore a region of stability in the chemical potential space.

This program will be of benefit to the theoretical and computational study of materials with 3 or more constituent species, particularly for the design of novel functional materials that are thermodynamically stable, and the generality of the present approach has clear advantages.

\section{Acknowledgment}

The authors acknowledge funding from EPSRC grant EP/IO1330X/1. D. O. S. is grateful to the Ramsay memorial trust and University College London for the provision of a Ramsay Fellowship. The authors also acknowledge the use of the UCL Legion High Performance Computing Facility (Legion@UCL) and associated support services, the IRIDIS cluster provided by the EPSRC funded Centre for Innovation (EP/K000144/1 and EP/K000136/1), and the HECToR supercomputer through membership of the UKs HPC Materials Chemistry Consortium, which is funded by EPSRC grant EP/F067496. We would like to thank M. R. Farrow and A. A. Sokol for useful discussions.

\section{Appendix A. Sorting routine}

In this appendix we describe the sorting algorithm used to extract all appropriate combinations from the set of linear equations derived from the conditions on the chemical potentials (see Sec. 3). We assume that there are $n$ unknowns, and $m$ linear equations, where $m \geq n$. The aim of the sorting algorithm is to extract all combinations of $n$ equations from the total $m$ (there will be ${ }^{m} C_{n}$ combinations).

The input to the routine is the matrix $M_{i j}$, which is $m \times(n+1)$ dimensional. Each row corresponds to a linear equation; the first $n$ columns are the coefficients of the $n$ unknowns, and the $n+1$-column is the right-handside of the linear equation. The output from the routine will be the ${ }^{m} C_{n}$ matrices $S_{i j}$, which are the $n \times n$ dimensional matrices of coefficients, and the vectors $v_{i}$, which are the $n$-dimensional corresponding vectors consisting 
of the right-hand-sides of the appropriate equations. $S$ and $v$ can then be used in a standard $L U$ decomposition and back-substitution approach [37] to determine the unknowns (i.e. to find the intersection points of the linear equations, if they exist).

The routine works by creating an array ival, which is $n$-dimensional. The elements in the array are initially set as the integers $1,2, \ldots, n$. The array is then used to construct $S$ and $v$. By sequentially changing the arrangement of the elements of the array (and allowing the array elements to adopt values up to $m$ ), all ${ }^{m} C_{n}$ combinations are extracted from the matrix $M$. The algorithm is shown in detail in Fig. A.7.

\section{References}

[1] A. Walsh, S. Chen, S.-H. Wei, X.-G. Gong, Adv. Energy Mater. 2 (2012) 400-409.

[2] D. M. Powell, M. T. Winkler, H. J. Choi, C. B. Simmons, D. B. Needleman, T. Buonassisi, Energy Environ. Sci. 5 (2012) 5874-5883.

[3] K. Ellmer, Nat. Photonics 6 (2012) 809-812.

[4] A. Walsh, A. B. Kehoe, D. J. Temple, G. W. Watson, D. O. Scanlon, Chem. Commun. 49 (2013) 448-450.

[5] G. Trimarchi, H. Peng, J. Im, A. J. Freeman, V. Cloet, A. Raw, K. R. Poeppelmeier, K. Biswas, S. Lany, A. Zunger, Phys. Rev. B 84 (2011) 165116.

[6] D. O. Scanlon, G. W. Watson, J. Mater. Chem. 22 (2012) 25236-25245.

[7] D. O. Scanlon, A. Walsh, Appl. Phys. Lett. 100 (2012) 251911.

[8] L. A. Burton, A. Walsh, J. Phys. Chem. C 116 (2012) 24262-24267.

[9] L. Lahourcade, N. C. Coronel, K. T. Delaney, S. K. Shukla, N. A. Spaldin, H. A. Atwater, Adv. Mater. (2013) n/a-n/a.

[10] J. P. Bosco, S. B. Demers, G. M. Kimball, N. S. Lewis, H. A. Atwater, J. Appl. Phys. 112 (2012) 093703. 
[11] N. Feldberg, B. Keen, J. D. Aldous, D. O. Scanlon, P. A. Stampe, R. J. Kennedy, R. J. Reeves, T. D. Veal, S. M. Durbin, in: Photovoltaic Specialists Conference (PVSC), 2012 38th IEEE, pp. 002524-002527.

[12] H. J. Kim, U. K. M. Kim, T. H. Kim, H. S. Mun, B.-G. Jeon, K. T. Hong, W.-J. Lee, C. Ju, K. H. Kim, K. Char, Appl. Phys. Express 5 (2012) 061102.

[13] D. O. Scanlon, P. D. C. King, R. P. Singh, A. de la Torre, S. McKeown Walker, G. Balakrishnan, F. Baumberger, C. R. A. Catlow, Adv. Mater. 24 (2012) 2154-2158.

[14] S. Chen, X. G. Gong, A. Walsh, S.-H. Wei, Appl. Phys. Lett. 94 (2009) 041903.

[15] H. Hiramatsu, T. Kamiya, T. Tohei, E. Ikenaga, T. Mizoguchi, Y. Ikuhara, K. Kobayashi, H. Hosono, J. Am. Chem. Soc. 132 (2010) 15060-15067.

[16] A. Zakutayev, J. Tate, H. A. S. Platt, D. A. Keszler, C. Hein, T. Mayer, A. Klein, W. Jaegermann, J. Appl. Phys. 107 (2010) 103713.

[17] D. C. Green, S. Glatzel, A. M. Collins, A. J. Patil, S. R. Hall, Adv. Mater. 24 (2012) 5767-5772.

[18] D. O. Scanlon, G. W. Watson, Chem. Mater. 21 (2009) 5435-5442.

[19] B. C. Melot, J.-M. Tarascon, Accounts Chem. Res. (2013) in press.

[20] R. Marom, S. F. Amalraj, N. Leifer, D. Jacob, D. Aurbach, J. Mater. Chem. 21 (2011) 9938-9954.

[21] K. C. Wincewicz, J. S. Cooper, J. Power Sources 1 (2005) 280-296.

[22] C. R. A. Catlow, Z. X. Guo, M. Miskufova, S. A. Shevlin, A. G. H. Smith, A. A. Sokol, A. Walsh, D. J. Wilson, S. M. Woodley, Philos. T. Roy. Soc. A 368 (2010) 3379-3456.

[23] S. M. Woodley, C. R. A. Catlow, Nat. Mater. 7 (2008) 937-946.

[24] S. Ping Ong, L. Wang, B. Kang, G. Ceder, Chem. Mater. 20 (2008) 1798-1807. 
[25] C. Persson, Y.-J. Zhao, S. Lany, A. Zunger, Phys. Rev. B 72 (2005) 035211.

[26] C. G. V. de Walle, J. Neugebauer, J. Appl. Phys. 95 (2004) 3851.

[27] D. O. Scanlon, G. W. Watson, J. Mater. Chem. 21 (2011) 3655-3663.

[28] D. O. Scanlon, G. W. Watson, The Journal of Physical Chemistry Letters 1 (2010) 3195-3199.

[29] G. Bergerhoff, I. D. Brown, Crystallographic Databases, International Union of Crystallography, F. H. Allen et al. (Hrsg.), Chester, 1987.

[30] S. B. Zhang, J. E. Northrup, Phys. Rev. Lett. 67 (1991) 2339-2342.

[31] K. Reuter, M. Scheffler, Phys. Rev. B 65 (2001) 035406.

[32] https://sourceforge.net/projects/cplap

[33] https://github.com/projects/cplap.git

[34] T. Williams, C. Kelley, many others, Gnuplot 4.4: an interactive plotting program, http://gnuplot.sourceforge.net, 2010.

[35] Mathematica, Version 8.0, Wolfram Research, Inc., Champaign, IL, USA, 2010.

[36] C. Kittel, H. Kroemer, Thermal Physics, chapter 9, W. H. Freeman and Company, second edition, 1980.

[37] W. H. Press, S. A. Teukolsky, W. T. Vetterling, B. P. Flannery, Numerical Recipes: The Art of Scientific Computing, Cambridge University Press, New York, NY, USA, third edition, 2007.

[38] L. Kaufman, H. Bernstein, Computer Calculation of Phase Diagrams with Special Reference to Refractory Metals, Academic Press, New York, NY, USA, 1970.

[39] N. Saunders, A. Miodownik (Eds.), CALPHAD (Calculation of Phase Diagrams): A Comprehensive Guide, Elsevier Science Inc., New York, NY, USA, 1998. 
[40] H. Lukas, S. G. Fries, B. Sundman, Computational Thermodynamics, The Calphad Method, Cambridge University Press, New York, NY, USA, 2007.

[41] D. O. Scanlon, Phys. Rev. B 87 (2013) 161201.

[42] M. Ernzerhof, G. E. Scuseria, J. Chem. Phys. 110 (1999) 5029-5036.

[43] C. Adamo, V. Barone, J. Chem. Phys. 110 (1999) 6158-6170.

[44] K. Ueda, H. Hosono, J. Appl. Phys. 91 (2002) 4768-4770.

[45] H. Hiramatsu, H. Kamioka, K. Ueda, H. Ohta, T. Kamiya, M. Hirano, H. Hosono, phys. status solidi A 203 (2006) 2800-2811.

[46] H. Hiramatsu, K. Ueda, H. Ohta, M. Hirano, M. Kikuchi, H. Yanagi, T. Kamiya, H. Hosono, Appl. Phys. Lett. 91 (2007) 012104.

[47] J. Heyd, G. E. Scuseria, M. Ernzerhof, J. Chem. Phys. 124 (2006) 219906. 


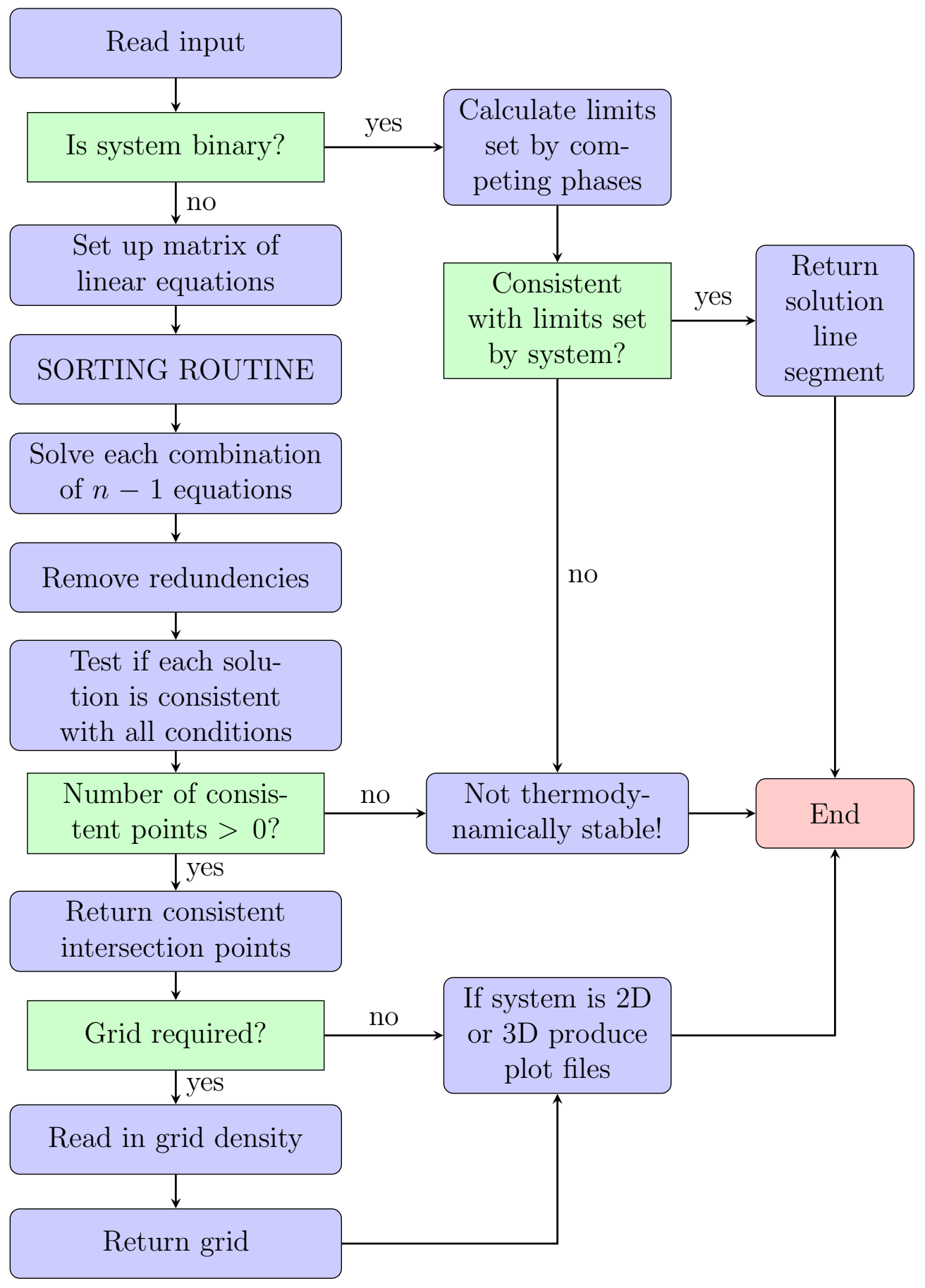

Figure 1: Flowchart of main algorithm. 


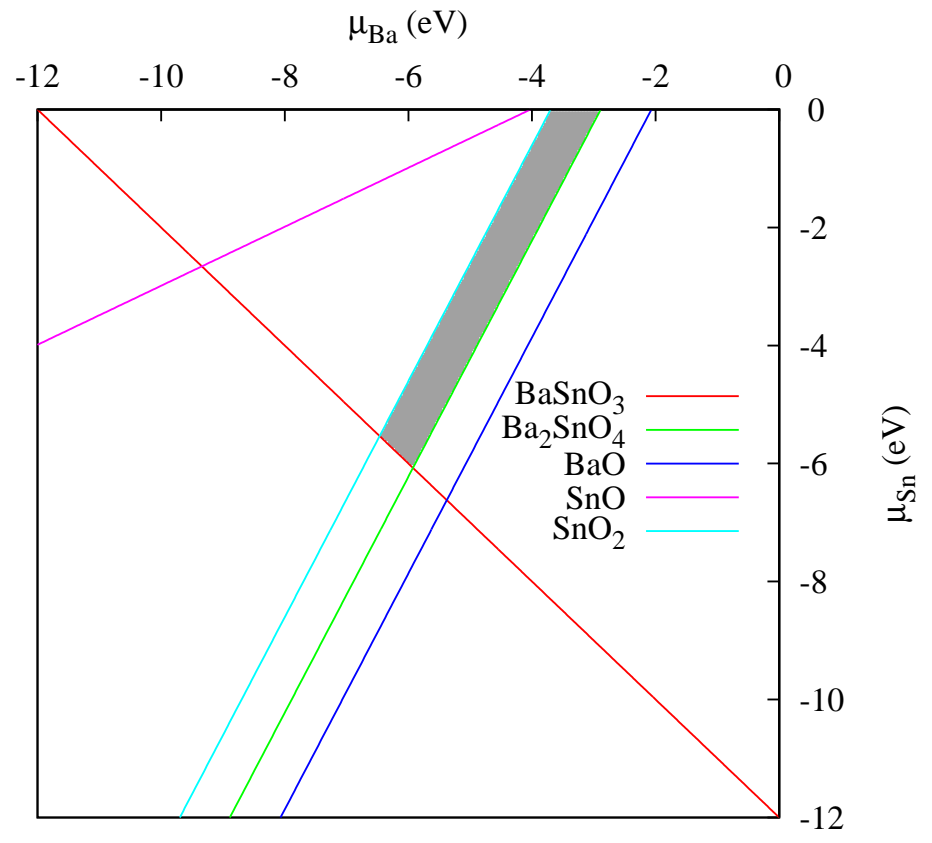

Figure 2: (Color online) Region of stability (shaded) for $\mathrm{BaSnO}_{3}$ in the 2D space spanned by $\mu_{\mathrm{Ba}}$ and $\mu_{\mathrm{Sn}}$. The (colored) lines indicate the limits imposed by the competing phases and the compound of interest. 


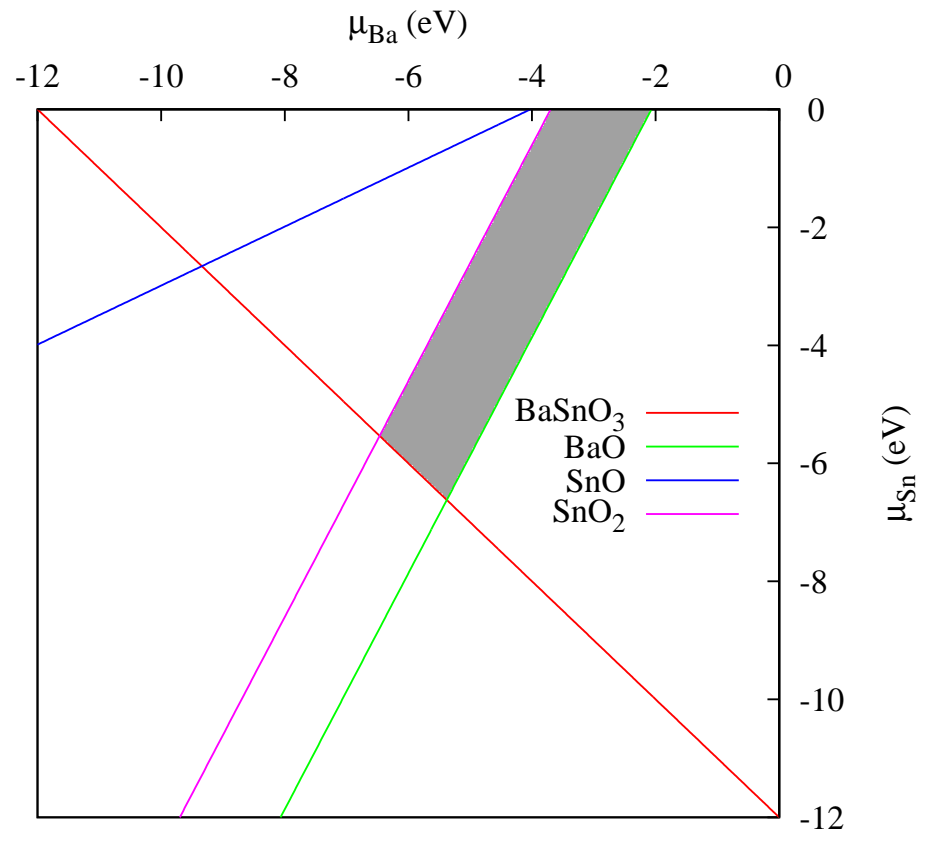

Figure 3: (Color online) Region of stability (shaded) for $\mathrm{BaSnO}_{3}$ in the 2D space spanned by $\mu_{\mathrm{Ba}}$ and $\mu_{\mathrm{Sn}}$ when the competing phase $\mathrm{Ba}_{2} \mathrm{SnO}_{4}$ is not taken into account. 


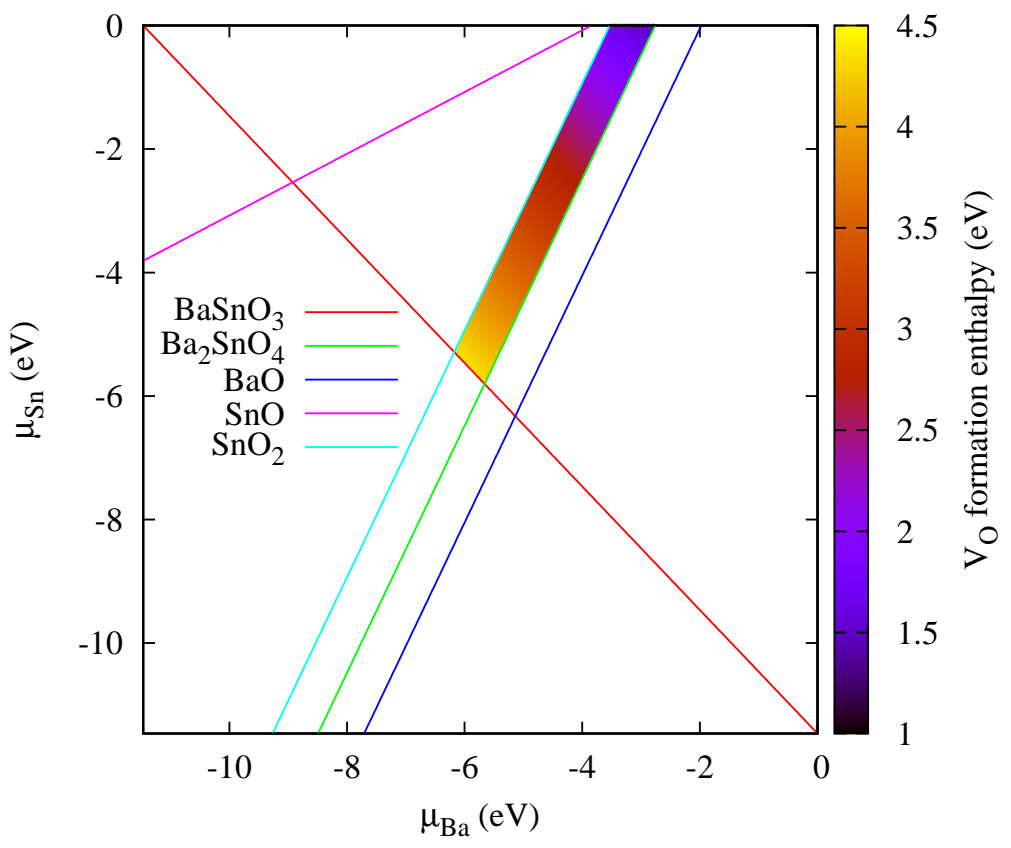

Figure 4: (Color online) Variation in $\mathrm{V}_{\mathrm{O}}$ formation enthalpy as a function of chemical potential, shown within the stability region for the formation of $\mathrm{BaSnO}_{3}$. The (colored) lines indicate the limits imposed by competing phases. 


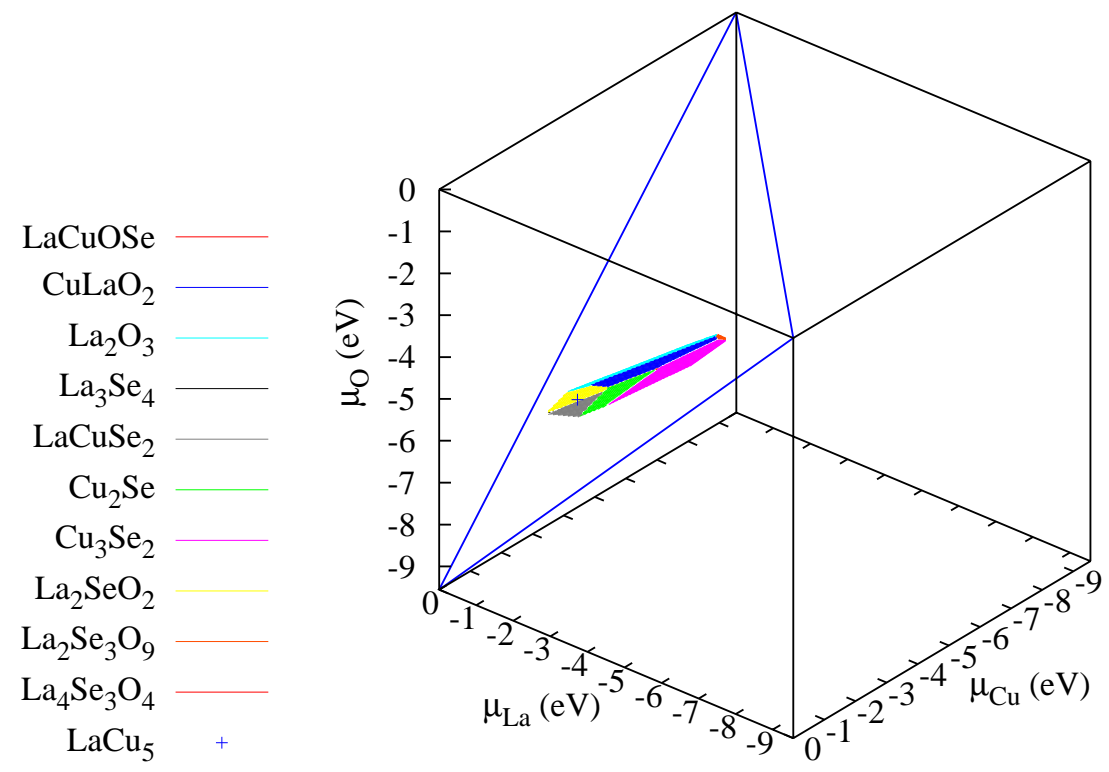

Figure 5: (Color online) Region of stability for LaCuOSe in the 3D space spanned by $\mu_{\mathrm{La}}$, $\mu_{\mathrm{Cu}}$ and $\mu_{\mathrm{O}}$. The thick (blue) lines indicate the boundary provided by the compound of interest (LaCuOSe). The (colored) surfaces indicate the limits imposed by the competing phases and the compound of interest. Surfaces parallel to the $z$-axis are represented by a cross at their mid-point. 


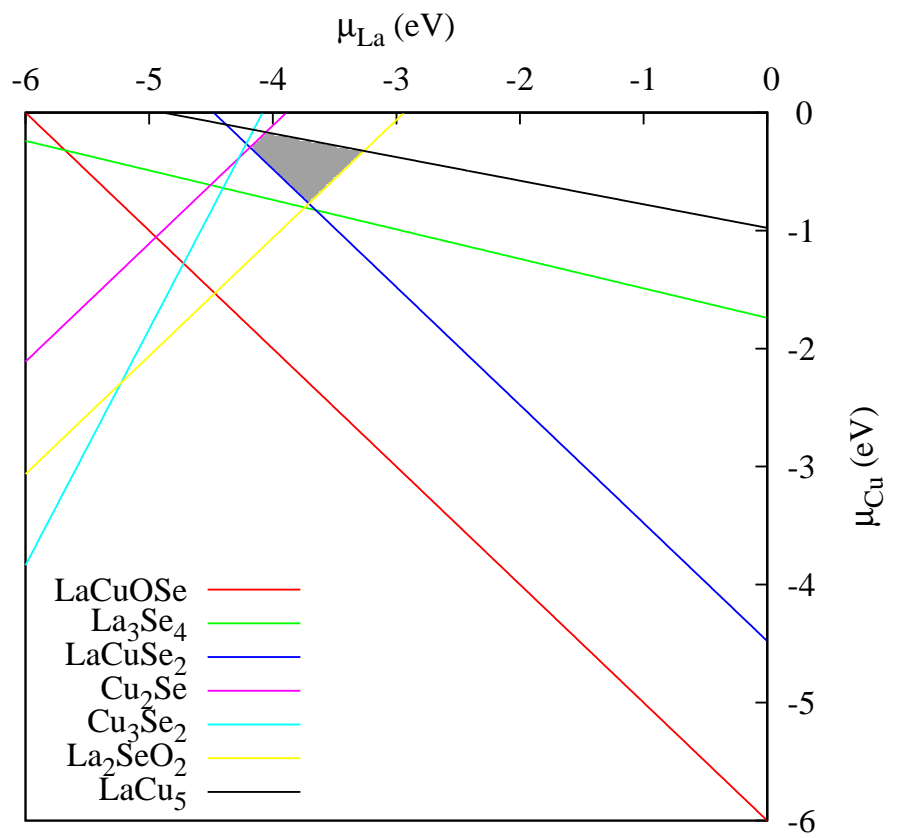

Figure 6: (Color online) Region of stability for LaCuOSe in O-poor growth conditions $\left(\mu_{\mathrm{O}}=-4 \mathrm{eV}\right)$. The chemical potential space is $2 \mathrm{D}$, spanned by $\mu_{\mathrm{La}}$ and $\mu_{\mathrm{Cu}}$. The region is effectively a 'slice' taken from the 3D stability region shown in Fig. 5. The (colored) lines indicate the limits imposed by the relevant competing phases and the compound of interest. 


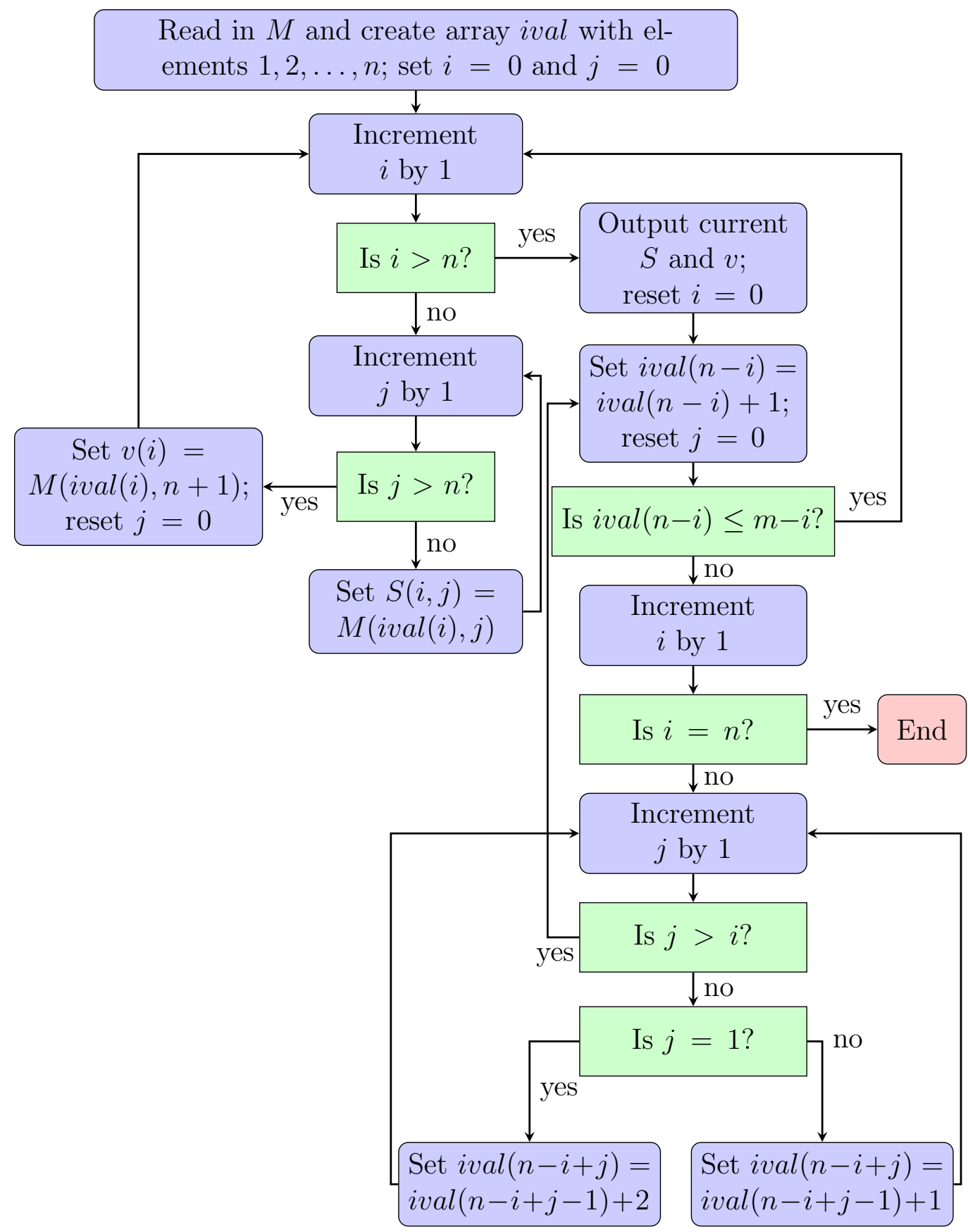

Figure A.7: Flowchart of sorting routine. 